This postprint is published in:

Annals of pure and applied Logic, July 2020, Volume 171, Issue 7, July 2020, art. 102806 (8 pp.)

DOI: 10.1016/j.apal.2020.102806

\title{
$M$-SEPARABLE SPACES OF FUNCTIONS ARE PRODUCTIVE IN THE MILLER MODEL
}

\author{
DUŠAN REPOVŠ AND LYUBOMYR ZDOMSKYY
}

\begin{abstract}
We prove that in the Miller model, every $M$-separable space of the form $C_{p}(X)$, where $X$ is metrizable and separable, is productively $M$ separable, i.e., $C_{p}(X) \times Y$ is $M$-separable for every countable $M$-separable $Y$.
\end{abstract}

\section{INTRODUCTION}

This paper is devoted to products of $M$-separable spaces. A topological space $X$ is said to be $M$-separable, if for every sequence $\left\langle D_{n}: n \in \omega\right\rangle$ of dense subsets of $X$, one can pick finite subsets $F_{n} \subset D_{n}$ so that $\bigcup_{n \in \omega} F_{n}$ is dense, see [3]. This notion was introduced in [21] where $M$-separable spaces of the form $C_{p}(X)$ were characterized. Here $C_{p}(X)$ is the set of all continuous functions $f: X \rightarrow \mathbb{R}$ with the topology inherited from the Tychonoff product $\mathbb{R}^{X}$. It is obvious that second-countable spaces (even spaces with a countable $\pi$-base) are $M$-separable. Our main result is the following

Theorem 1.1. In the Miller model, the product of any two $M$-separable spaces is $M$-separable, provided that all dense subspaces of this product are separable and one of the spaces is of the form $C_{p}(Z)$ for some Tychonoff space $Z$.

In particular, if $Y$ is a countable $M$-separable space and $X=C_{p}(Z)$ is $M$ separable for some second-countable $Z$, then $X \times Y$ is $M$-separable.

By the Miller model we standardly mean a forcing extension of a model of GCH by adding a generic filter for an iteration with countable supports of length $\omega_{2}$ of the poset introduced by Miller in [15]. We give more details about this poset in the next section. One of the key properties of this poset is the inequality $\mathfrak{u}<\mathfrak{g}$ proved in $[6,9]$, see [5] for more information on cardinal characteristics of the reals. In particular, an equivalent form of this inequality established in [12] will be crucial for our proof of Lemma 2.4.

Let us recall that a topological space $X$ is said to have the Menger property (or, alternatively, is a Menger space) if for every sequence $\left\langle\mathcal{U}_{n}: n \in \omega\right\rangle$ of open covers of $X$ there exists a sequence $\left\langle\mathcal{V}_{n}: n \in \omega\right\rangle$ such that each $\mathcal{V}_{n}$ is a finite subfamily of $\mathcal{U}_{n}$ and the collection $\left\{\cup \mathcal{V}_{n}: n \in \omega\right\}$ is a cover of $X$. This property was introduced by Hurewicz, and the current name (the Menger property) is used because Hurewicz proved in [11] that for metrizable spaces his property is equivalent to a certain property of a base considered earlier by Menger in [14]. The Menger property is central to the study of the $M$-separability of function

2010 Mathematics Subject Classification. Primary: 03E35, 54D20. Secondary: 54C50, $03 \mathrm{E} 05$.

Key words and phrases. $M$-separable, Miller forcing, Menger space, spaces of functions. 
spaces: For a Tychonoff space $X, C_{p}(X)$ is $M$-separable if and only if all finite powers of $X$ are Menger and $X$ admits a weaker separable metrizable topology, see [4, Theorem 2.9] or [21, Theorem 35]. Let us also note that by the main result of [24], all finite powers of $C_{p}(X)$ are hereditarily separable if all finite powers of $X$ are hereditarily Lindelöf. In particular, $C_{p}(Z)$ is hereditarily separable for second countable spaces $Z$.

Our paper is a further development of the ideas in $[18,19,23]$. However, the proof of Theorem 1.1 is conceptually different from those in these three papers, since here we have to analyze the local structure of spaces of functions in the Miller model. Also, unlike in [23], we were unable to achieve the optimal result (which would be the consistency of the preservation of $M$-separability by finite products of countable spaces), and affirmative answers to any of the last two items in Question 1.2 would fill in this gap by Lemma 2.5.

The main result of [23] states that in the Miller model, the product of any two second-countable spaces with the Menger property is Menger. Thus in this model the characterization mentioned above yields that for any two second-countable spaces $Z_{0}, Z_{1}$, if $C_{p}\left(Z_{0}\right)$ and $C_{p}\left(Z_{1}\right)$ are $M$-separable, then so is $C_{p}\left(Z_{0}\right) \times C_{p}\left(Z_{1}\right)$. Thus it is worth mentioning here that there are countable $M$-separable spaces which cannot be embedded into $M$-separable spaces of the form $C_{p}(Z)$, and hence Theorem 1.1 indeed covers more cases of $M$-separable spaces as the main result of [23] combined with the characterization in [4, 21]. The easiest example of such a space is the Fréchet-Urysohn fan $S_{\omega}$, i.e., the factor space of the product $\omega \times(\{0\} \cup\{1 / n: n \in \omega\}) \subset \mathbb{R}^{2}$ obtained by identifying all points in $\omega \times\{0\}$. It is obviously $M$-separable, and it fails to have the countable fan tightness introduced in [1], whereas every $M$-separable space of the form $C_{p}(Z)$ has countable fan tightness by [4, Corollary 2.10] and the latter property is hereditary.

On the other hand, there are many consistent examples under $\mathrm{CH}$ and weakenings thereof of countable $M$-separable spaces with non- $M$-separable products, see, e.g., $[2,17]$. As it was demonstrated in $[16, \S 6]$, in all cases when such a non-preservation result is known, one can obtain it by using spaces of the form $C_{p}(Z)$, which is a yet another motivation behind Theorem 1.1.

Theorem 1.1 seems to be the best known approximation towards the answer to the first item of following question which is central in this area. It was first asked in [4] and then repeated in several other papers. We refer the reader to Definition 2.1 for the notions appearing in the last two items.

Question 1.2. (1) Is it consistent that the product of two countable $M$ separable spaces is $M$-separable? Does this statement hold in the Miller model? Does it follow from $\mathfrak{u}<\mathfrak{g}$ ?

(2) In the Miller model, does every countable $M$-separable space $X$ have a point $x$ (equivalently, densely many points $x$ ) such that $\zeta(X, x) \leq \omega_{1}$ ?

(3) In the Miller model, does every countable $M$-separable space $X$ have property $(\dagger)$ ?

\section{Proof of Theorem 1.1}

We divide the proof of Theorem 1.1 into a sequence of auxiliary statements. More precisely, it will follow immediately from Lemmata 2.3, 2.5, 2.6, and 2.7. 
Definition 2.1. (1) A family $\mathcal{U}$ of open subsets of a space $X$ is called centered, if $\cap \mathcal{V} \neq \emptyset$ for any $\mathcal{V} \in[\mathcal{U}]^{<\omega}$.

(2) A topological space $\langle X, \tau\rangle$ is said to have property (†) if for every family $\mathrm{R}$ of size $\omega_{1}$ of functions $R$ assigning to each countable centered family $\mathcal{U}$ of open subsets of $X$ a sequence $R(\mathcal{U}) \in\left([X]^{<\omega} \backslash\{\emptyset\}\right)^{\omega}$ such that

$$
\forall U \in \mathcal{U} \forall^{\infty} n \in \omega(R(\mathcal{U})(n) \subset U),
$$

there $^{1}$ exists $U \in\left[[\tau \backslash\{\emptyset\}]^{\omega}\right]^{\omega_{1}}$ consisting of countable centered families such that for all $O \in \tau \backslash\{\emptyset\}$ there exists $\mathcal{U} \in \mathrm{U}$ such that for every $R \in \mathrm{R}$ there exists $n \in \omega$ with the property $R(\mathcal{U})(n) \subset O$.

(3) For a topological space $X$ and $x \in X$ we denote by $\zeta(X, x)$ the minimal cardinality $\kappa$ such that for every sequence $\left\langle A_{n}: n \in \omega\right\rangle$ such that $x \in \bar{A}_{n}$ for all $n$, there exists a sequence $\left\langle\left\langle K_{n}^{\alpha}: n \in \omega\right\rangle: \alpha<\kappa\right\rangle$ such that $K_{n}^{\alpha} \in\left[A_{n}\right]^{<\omega}$ for all $n, \alpha$, and for every open $U \ni x$ there exists $\alpha \in \kappa$ such that $U \cap K_{n}^{\alpha} \neq \emptyset$ for all $n \in \omega$.

(4) For a topological space $X$ we denote by $\zeta(X)$ the cardinal $\sup \{\zeta(X, x)$ : $x \in X\}$.

Spaces $X$ with $\zeta(X) \leq \omega$ are exactly the spaces which are weakly Fréchet in the strict sense in the terminology of $[3,20]$.

In order to prove Theorem 1.1 we need to recall some details related to the Miller forcing. By a Miller tree we understand a subtree $T$ of $\omega^{<\omega}$ consisting of increasing finite sequences such that the following conditions are satisfied:

- Every $t \in T$ has an extension $s \in T$ which splits in $T$, i.e., there are more than one immediate successors of $s$ in $T$;

- If $s$ is splitting in $T$, then it has infinitely many successors in $T$.

The Miller forcing is the collection $\mathbb{M}$ of all Miller trees ordered by inclusion, i.e., smaller trees carry more information about the generic. This poset was introduced in [15] and has since then found numerous applications see, e.g., [9]. We denote by $\mathbb{P}_{\alpha}$ an iteration of length $\alpha$ of the Miller forcing with countable support. If $G$ is $\mathbb{P}_{\beta}$-generic and $\alpha<\beta$, then we denote the intersection $G \cap \mathbb{P}_{\alpha}$ by $G_{\alpha}$.

For a Miller tree $T$ we shall denote by $\operatorname{Split}(T)$ the set of all splitting nodes of $T$. For a node $t$ in a Miller tree $T$ we denote by $T_{t}$ the set $\{s \in T: s$ is compatible with $t\}$. It is clear that $T_{t}$ is also a Miller tree. The stem of a Miller tree $T$ is the shortest $t \in \operatorname{Split}(T)$. We denote the stem of $T$ by $T\langle 0\rangle$. If $T_{1} \leq T_{0}$ and $T_{1}\langle 0\rangle=T_{0}\langle 0\rangle$, then we write $T_{1} \leq^{0} T_{0}$.

The following lemma can be proved by an almost verbatim repetition of the proof of [13, Lemma 14], see also [23, §2] for a more general form. Here by a real we mean a subset of $\omega$.

Lemma 2.2. Let $\dot{x}$ be a $\mathbb{P}_{\omega_{2}}$-name for a real and $p \in \mathbb{P}_{\omega_{2}}$. Then there exist $p^{\prime} \leq p$ such that $p^{\prime}(0) \leq 0$ that for each $N \in \omega, s \in \operatorname{Split}\left(p^{\prime}(0)\right)$, and for all but finitely many immediate successors $t$ of $s$ in $p^{\prime}(0)$ we have

$$
\left(p^{\prime}(0)\right)_{t}{ }^{\prime} p^{\prime}\left\lceil\left[1, \omega_{2}\right) \Vdash \exists u \in U_{s}(u \cap N=\dot{x} \cap N) .\right.
$$

\footnotetext{
${ }^{1}$ Here $\forall^{\infty}$ means "for all but finitely many".
} 
A subset $C$ of $\omega_{2}$ is called an $\omega_{1}-c l u b$ if it is unbounded and for every $\alpha \in \omega_{2}$ of cofinality $\omega_{1}$, if $C \cap \alpha$ is cofinal in $\alpha$ then $\alpha \in C$.

The following lemma will be the key part of the proof of Theorem 1.1.

Lemma 2.3. In the Miller model every countable space $X$ such that $\{x \in X$ : $\left.\zeta(X, x) \leq \omega_{1}\right\}$ is dense in $X$, has property $(\dagger)$.

Proof. We work in $V\left[G_{\omega_{2}}\right]$, where $G_{\omega_{2}}$ is $\mathbb{P}_{\omega_{2}}$-generic and $\mathbb{P}_{\omega_{2}}$ is the iteration of length $\omega_{2}$ with countable supports of the Miller forcing. Let us write $X$ in the form $\langle\omega, \tau\rangle$ and let $\mathrm{R}=\left\{R_{\alpha}: \alpha<\omega_{1}\right\}$ be such as in the definition of $(\dagger)$. By a standard argument (see, e.g., the proof of [8, Lemma 5.10]) there exists an $\omega_{1}$-club $C \subset \omega_{2}$ such that for every $\alpha \in C$ the following conditions hold:

(i) $\tau \cap V\left[G_{\alpha}\right] \in V\left[G_{\alpha}\right]$ and for every $x \in \omega$ and every sequence $\left\langle A_{n}: n \in\right.$ $\omega\rangle \in V\left[G_{\alpha}\right]$ of subsets of $\omega$ containing $x$ in their closure, there exists $\left\langle\left\langle K_{n}^{\alpha}: n \in \omega\right\rangle: \alpha<\omega_{1}\right\rangle \in V\left[G_{\alpha}\right]$ such as Definition 2.1(3);

(ii) $\left\{R_{\alpha}(\mathcal{U}): \alpha \in \omega_{1}, \mathcal{U} \in\left[\tau \cap V\left[G_{\alpha}\right]\right]^{\omega} \cap V\left[G_{\alpha}\right]\right.$ is centered $\} \in V\left[G_{\alpha}\right]$;

(iii) For every $A \in \mathcal{P}(\omega) \cap V\left[G_{\alpha}\right]$ the interior $\operatorname{Int}(A)$ also belongs to $V\left[G_{\alpha}\right]$.

Standardly, there is no loss of generality in assuming that $0 \in C$. We claim that

$$
\mathcal{U}:=\left\{\mathcal{U} \in[\tau \backslash\{\emptyset\}]^{\omega} \cap V: \mathcal{U} \text { is centered }\right\}
$$

is a witness for $\langle\omega, \tau\rangle$ satisfying $(\dagger)$. Suppose, contrary to our claim, that there exists $A \in \tau \backslash\{\emptyset\}$ such that for every $\mathcal{U} \in \mathrm{U}$ there exists $\alpha \in \omega_{1}$ such that $R_{\alpha}(\mathcal{U})(n) \not \subset A$ for all $n \in \omega$. Let $\dot{A}$ be a $\mathbb{P}_{\omega_{2}}$-name for $A$ and $p \in \mathbb{P}_{\omega_{2}}$ a condition forcing the above statement. Without loss of generality, we may assume that there exists $N \in \omega$ such that $\zeta(X, N) \leq \omega_{1}$ and $p \Vdash N \in \dot{A}$.

Applying Lemma 2.2 to $\dot{x}:=\dot{A}$, we get a condition $p^{\prime} \leq p$ such that $p^{\prime}(0) \leq^{0}$ $p(0)$, and a finite set $\mathcal{U}_{s} \subset \mathcal{P}(\omega)$ for every $s \in \operatorname{Split}\left(p^{\prime}(0)\right)$, such that for each $n \in \omega, s \in \operatorname{Split}\left(p^{\prime}(0)\right)$, and for all but finitely many immediate successors $t$ of $s$ in $p^{\prime}(0)$ we have

$$
p^{\prime}(0)_{t}{ }^{\wedge} p^{\prime} \uparrow\left[1, \omega_{2}\right) \Vdash \exists U \in \mathcal{U}_{s}(\dot{A} \cap n=U \cap n) .
$$

Of course, any $p^{\prime \prime} \leq p^{\prime}$ also has the above property with the same $\mathcal{U}_{s}$ 's. However, the stronger $p^{\prime \prime}$ is, the more elements of $\mathcal{U}_{s}$ might play no role any more. Therefore throughout the rest of the proof we shall call $U \in \mathcal{U}_{s}$ void for $p^{\prime \prime} \leq p^{\prime}$ and $s \in \operatorname{Split}\left(p^{\prime \prime}(0)\right)$, if there exists $n \in \omega$ such that for all but finitely many immediate successors $t$ of $s$ in $p^{\prime \prime}(0)$ there is $n o q \leq p^{\prime \prime}(0)_{t}{ }^{\wedge} p^{\prime \prime} \uparrow\left[1, \omega_{2}\right)$ with the property $q \Vdash \dot{A} \cap n=U \cap n$. Note that for any $p^{\prime \prime} \leq p^{\prime}$ and $s \in \operatorname{Split}\left(p^{\prime \prime}(0)\right)$ there exists $U \in \mathcal{U}_{s}$ which is non-void for $p^{\prime \prime}, s$. Two cases are possible.

Case $a$ ) For every $p^{\prime \prime} \leq p^{\prime}$ there exists $s \in \operatorname{Split}\left(p^{\prime \prime}(0)\right)$ and a non-void $U \in \mathcal{U}_{s}$ for $p^{\prime \prime}, s$ such that $N \in \operatorname{Int}(U)$. Let $\mathcal{U}$ be the collection of $\operatorname{Int}(U)$ for all $U$ as above. It follows from the above that $p^{\prime}$ forces that there exists $\alpha \in \omega_{1}$ such that $R_{\alpha}(\mathcal{U})(n) \not \subset \dot{A}$ for all $n \in \omega$. Passing to a stronger condition if necessary, we may additionally assume that $p^{\prime}$ decides $\alpha$.

Fix a non-void $U$ for $p^{\prime}, s$, where $s \in \operatorname{Split}\left(p^{\prime}(0)\right)$, such that $N \in \operatorname{Int}(U)$ (and hence $\operatorname{Int}(U) \in \mathcal{U})$. It follows from the above that there exists $m$ such that $R_{\alpha}(\mathcal{U})(k) \subset \operatorname{Int}(U)$ for all $k \geq m$. Let $n \in \omega$ be such that $R_{\alpha}(\mathcal{U})(m) \subset n$. By the definition of being non-void, there are infinitely many immediate successors $t$ of $s$ in $p^{\prime}(0)$ for which there exists $q_{t} \leq p^{\prime}(0)_{t}{ }^{\wedge} p^{\prime} \uparrow\left[1, \omega_{2}\right)$ with the property $q_{t} \Vdash \dot{A} \cap n=U \cap n$. Then for any $q_{t}$ as above we have that $q_{t}$ forces $R_{\alpha}(\mathcal{U})(m) \subset$ 
$\dot{A}$ because $R_{\alpha}(\mathcal{U})(m) \subset U \cap n$, which contradicts the fact that $q_{t} \leq p^{\prime}$ and $p^{\prime} \Vdash R_{\alpha}(\mathcal{U})(m) \not \subset \dot{A}$.

Case $b)$ There exists $p^{\prime \prime} \leq p^{\prime}$ such that for all $s \in \operatorname{Split}\left(p^{\prime \prime}(0)\right)$, every $U \in \mathcal{U}_{s}$ with $N \in \operatorname{Int}(U)$ is void for $p^{\prime \prime}, s$. Note that this implies that every $U \in \mathcal{U}_{s}$ with $N \in \operatorname{Int}(U), U$ is void for $q, s$ for all $q \leq p^{\prime \prime}$ and $s \in \operatorname{Split}(q(0))$. Let $\left\langle D_{k}: k \in \omega\right\rangle \in V$ be a sequence of subsets of $\omega$ such that

$$
\left\{D_{k}: k \in \omega\right\}=\left\{\omega \backslash U: U \in \bigcup_{s \in \operatorname{Split}\left(p^{\prime \prime}(0)\right)} \mathcal{U}_{s}, N \notin \operatorname{Int}(U)\right\} .
$$

Item $(i)$ above yields a sequence $\left\langle\left\langle K_{k}^{\alpha}: k \in \omega\right\rangle: \alpha<\omega_{1}\right\rangle \in V$ such that $K_{k}^{\alpha} \in\left[D_{k}\right]^{<\omega}$ for all $k$, and for every neighborhood $O \in \tau$ of $N$ there exists $\alpha \in \omega_{1}$ such that $K_{k}^{\alpha} \cap O \neq \emptyset$ for all $k \in \omega$. Let $p^{(3)} \leq p^{\prime \prime}$ decide $\alpha$ which has the property stated above for $\dot{A}$. Fix $U \in \mathcal{U}_{p^{(3)}(0)\langle 0\rangle}$ non-void for $p^{(3)}, p^{(3)}(0)\langle 0\rangle$. Then $N \notin \operatorname{Int}(U)$ by the choice of $p^{\prime \prime}$ and hence there exists $k$ such that $\omega \backslash U=D_{k}$. It follows that $K_{k}^{\alpha} \cap U=\emptyset$ because $K_{k}^{\alpha} \subset D_{k}$. On the other hand, since $U$ is nonvoid for $p^{(3)}, p^{(3)}(0)\langle 0\rangle$, for $n=\max K_{k}^{\alpha}+1$ we can find infinitely many immediate successors $t$ of $p^{(3)}(0)\langle 0\rangle$ in $p^{(3)}(0)$ for which there exists $q_{t} \leq p^{(3)}(0)_{t} \wedge p^{(3)} \uparrow\left[1, \omega_{2}\right)$ forcing $\dot{A} \cap n=U \cap n$. Then any such $q_{t}$ forces $K_{k}^{\alpha} \cap \dot{A}=\emptyset$ (because $K_{k}^{\alpha} \subset n$ and $\left.K_{k}^{\alpha} \cap U=\emptyset\right)$, contradicting the fact that $p^{(3)} \geq q_{t}$ and $p^{(3)} \Vdash K_{k}^{\alpha} \cap \dot{A} \neq \emptyset$ for all $k$.

Contradictions obtained in cases $a$ ) and $b$ ) above imply that $\mathrm{U}$ is a witness for $\langle\omega, \tau\rangle$ having $(\dagger)$, which completes the proof of Lemma 2.3.

It is well-known [9] that in the Miller model there exists an ultrafilter $\mathcal{F}$ generated by $\omega_{1}$-many sets, say $\left\{F_{\alpha}: \alpha \in \omega_{1}\right\}$. It plays an important role in the proof of the following

Lemma 2.4. In the Miller model, for every $M$-separable space $X$ and every decreasing sequence $\left\langle D_{n}: n \in \omega\right\rangle$ of countable dense subsets of $X$, there exists a sequence $\left\langle\left\langle K_{n}^{\alpha}: n \in \omega\right\rangle: \alpha \in \omega_{1}\right\rangle$ such that

(1) $K_{n}^{\alpha} \in\left[D_{n}\right]^{<\omega}$ for all $n \in \omega$ and $\alpha \in \omega_{1}$; and

(2) for every open non-empty $O \subset X$, there exists $\alpha \in \omega_{1}$ such that $O \cap K_{n}^{\alpha} \neq$ $\emptyset$ for all $n \in \omega$.

Proof. Let us write $D_{n}$ in the form $\left\{d_{k}^{n}: k \in \omega\right\}$ and fix an increasing function $f \in \omega^{\omega}$ such that for every open non-empty $O \subset X$ there are infinitely many $n \in \omega$ such that $O \cap\left\{d_{k}^{n}: k \leq f(n)\right\} \neq \emptyset$. (This is possible due to the $M$-separability of $X$.) Let us denote by $U_{O}$ the set of all such $n$. By [12, Theorem 10] combined with $[6 \text {, Theorems } 1,2]^{2}$, for the family $\mathcal{U}=\left\{U_{O}: O\right.$ is an open non-empty subset of $X\}$ there exists an increasing sequence $\left\langle m_{i}: i \in \omega\right\rangle \in \omega^{\omega}$ such that one of the following options takes place:

- For every $O$, the set $\bigcup\left\{\left[m_{i}, m_{i+1}\right): U_{O} \cap\left[m_{i}, m_{i+1}\right) \neq \emptyset\right\}$ belongs to $\mathcal{F}$; or

- For every $A \in[\omega]^{\omega}$, there exists $O$ such that $U_{O} \subset^{*} \bigcup\left\{\left[m_{i}, m_{i+1}\right): i \in A\right\}$.

\footnotetext{
${ }^{2}$ As noted by the referee, these results from $[6,12]$ only give certain finite-to-one function. However, it is rather standard and not too difficult to derive from this function an increasing sequence $\left\langle m_{i}: i \in \omega\right\rangle \in \omega^{\omega}$ with the properties we need in this proof.
} 
Suppose that the second option takes place and let $\mathcal{A} \subset[\omega]^{\omega}$ be an infinite (and hence uncountable) maximal almost disjoint family. For every $A \in \mathcal{A}$, fix an open non-empty subset $O(A)$ of $X$ such that $U_{O(A)} \subset^{*} \bigcup\left\{\left[m_{i}, m_{i+1}\right): i \in A\right\}$ and note that this implies $\left|U_{O(A)} \cap U_{O\left(A^{\prime}\right)}\right|<\omega$ for any distinct $A, A^{\prime} \in \mathcal{A}$. On the other hand, since $X$ is separable and $\mathcal{A}$ is uncountable, there are distinct $A, A^{\prime} \in \mathcal{A}$ such that $O(A) \cap O\left(A^{\prime}\right) \neq \emptyset$, and hence $U_{O(A) \cap O\left(A^{\prime}\right)}$ is infinite, contradicting the fact that $U_{O(A) \cap O\left(A^{\prime}\right)} \subset U_{O(A)} \cap U_{O\left(A^{\prime}\right)}$ and the latter intersection is finite.

Thus the first option must take place. For every $\alpha \in \omega_{1}$ and $n \in \omega$ let $i_{\alpha, n}$ be the minimal number $i$ such that $m_{i} \geq n$ and $\left[m_{i}, m_{i+1}\right) \cap F_{\alpha} \neq \emptyset$. We claim that the sequences

$$
\left\langle K_{n}^{\alpha}=\left\{d_{k}^{l}: l \in\left[m_{i_{\alpha, n}}, m_{i_{\alpha, n}+1}\right), k \leq f(l)\right\}: n \in \omega\right\rangle
$$

are as required. Indeed, given $O$, find $\alpha$ such that $F_{\alpha} \subset \bigcup\left\{\left[m_{i}, m_{i+1}\right): U_{O} \cap\right.$ $\left.\left[m_{i}, m_{i+1}\right) \neq \emptyset\right\}$. Now for every $n \in \omega$ we have

$$
K_{n}^{\alpha} \cap O=\left\{d_{k}^{l}: l \in\left[m_{i_{\alpha, n}}, m_{i_{\alpha, n}+1}\right), k \leq f(l)\right\} \cap O,
$$

and the latter intersection is non-empty because $\left[m_{i_{\alpha, n}}, m_{i_{\alpha, n}+1}\right) \cap F_{\alpha} \neq \emptyset$, hence also $\left[m_{i_{\alpha, n}}, m_{i_{\alpha, n}+1}\right) \cap U_{O} \neq \emptyset$, and thus for every $l \in\left[m_{i_{\alpha, n}}, m_{i_{\alpha, n}+1}\right) \cap U_{O}$ we have $O \cap\left\{d_{k}^{l}: k \leq f(l)\right\} \neq \emptyset$. This completes the proof of Lemma 2.4.

There is a natural linear preorder $\leq_{\mathcal{F}}$ on $\omega^{\omega}$ associated to $\mathcal{F}$ defined as follows: $x \leq_{\mathcal{F}} y$ if and only if $\{n \in \omega: x(n) \leq y(n)\} \in \mathcal{F}$. By [7, Theorem 3.1], in the Miller model, for every $X \subset \omega^{\omega}$ of size $\omega_{1}$ there exists $b \in \omega^{\omega}$ such that $x \leq_{\mathcal{F}} b$ for all $x \in X$. As an easy consequence thereof we get the following fact: Suppose that $\left\langle D_{n}: n \in \omega\right\rangle$ is a sequence of countable sets and $A_{\alpha, n} \in\left[D_{n}\right]^{<\omega}$ for all $\alpha \in \omega_{1}$ and $n \in \omega$. Then there exists a sequence $\left\langle A_{n}: n \in \omega\right\rangle$ such that $A_{n} \in\left[D_{n}\right]<\omega$ for all $n$, and $\left\{n: A_{\alpha, n} \subset A_{n}\right\} \in \mathcal{F}$ for all $\alpha \in \omega_{1}$.

Lemma 2.5. In the Miller model, suppose that $|X|=|Y|=\omega, X$ satisfies ( $\dagger$ ), and $Y$ is $M$-separable. Then $X \times Y$ is $M$-separable.

Proof. Let $\left\langle D_{n}: n \in \omega\right\rangle$ be a sequence of dense subsets of $X \times Y$. By [10, Lemma 2.1], there is no loss of generality in assuming that $D_{n+1} \subset D_{n}$ for all $n$. Given an open non-empty subset $U$ of $X$, for every $n \in \omega$ set $D_{n}^{U}=\{y \in$ $\left.Y: \exists x \in U\left(\langle x, y\rangle \in D_{n}\right)\right\}$ and note that $D_{n}^{U}$ is dense in $Y$. Given a countable centered family $\mathcal{U}$ of open subsets of $X$, fix a decreasing sequence $\left\langle U_{\mathcal{U}, n}: n \in \omega\right\rangle$ of open subsets of $X$ such that for every $U \in \mathcal{U}$, there exists $n \in \omega$ such that $U_{\mathcal{U}, n} \subset U$. By Lemma 2.4 there exists a sequence

$$
\left\langle\left\langle L_{n}^{\alpha, \mathcal{U}}: n \in \omega\right\rangle: \alpha \in \omega_{1}\right\rangle
$$

such that $L_{n}^{\alpha, \mathcal{U}} \in\left[D_{n}^{U_{\mathcal{U}, n}}\right]^{<\omega}$ for all $n, \alpha$, and for every open non-empty $V \subset Y$, there exists $\alpha$ such that $L_{n}^{\alpha, \mathcal{U}} \cap V \neq \emptyset$ for all $n$. Let us find $K_{n}^{\alpha, \mathcal{U}} \in\left[U_{\mathcal{U}, n}\right]^{<\omega}$ such that for every $y \in L_{n}^{\alpha, \mathcal{U}}$, there exists $x \in K_{n}^{\alpha, \mathcal{U}}$ such that $\langle x, y\rangle \in D_{n}$. For every $\alpha, \beta \in \omega_{1}$ and $n \in \omega$, set $R_{\alpha, \beta}(\mathcal{U})(n)=K_{\min \left(F_{\beta} \backslash n\right)}^{\alpha, \mathcal{U}}$. Note that $\mathrm{R}=\left\{R_{\alpha, \beta}: \alpha, \beta \in\right.$ $\left.\omega_{1}\right\}$ is such as in the definition of $(\dagger)$ because $K_{n}^{\alpha, \mathcal{U}} \subset U$ for all $U \in \mathcal{U}$ and all but finitely many $n \in \omega$. It follows that there exists a family $U$ of countable centered families $\mathcal{U}$ of open subsets of $X$ of size $|\mathrm{U}|=\omega_{1}$, and such that for every open non-empty $O \subset X$, there exists $\mathcal{U} \in \mathrm{U}$ such that for all $\alpha, \beta \in \omega_{1}$, there exists 
$n \in F_{\beta}$ with the property $K_{n}^{\alpha, \mathcal{U}} \subset O$. Since $\mathcal{F}$ is an ultrafilter, it follows that for all $\alpha \in \omega_{1}$, there exists $\xi \in \omega_{1}$ with the property $K_{n}^{\alpha, \mathcal{U}} \subset O$ for all $n \in F_{\xi}$.

Since $|\mathcal{U}|=\omega_{1}$, there exists a sequence $\left\langle M_{n}: n \in \omega\right\rangle$ such that $M_{n} \in\left[D_{n}\right]^{<\omega}$ and for every $\mathcal{U} \in \mathrm{U}$ and $\alpha, \beta \in \omega_{1}$, we have

$$
\left\{n \in \omega: M_{n} \supset\left(K_{n}^{\alpha, \mathcal{U}} \times L_{n}^{\alpha, \mathcal{U}}\right) \cap D_{n}\right\} \in \mathcal{F} .
$$

We claim that $\bigcup_{n \in \omega} M_{n}$ is dense in $X \times Y$. Indeed, let us fix an open non-empty subset of $X \times Y$ of the form $O \times V$ and find $\mathcal{U} \in \mathrm{U}$ as above. Let $\alpha$ be such that $L_{n}^{\alpha, \mathcal{U}} \cap V \neq \emptyset$ for all $n \in \omega$. Pick $\beta \in \omega_{1}$ such that

$$
F_{\beta} \subset\left\{n \in \omega: M_{n} \supset\left(K_{n}^{\alpha, \mathcal{U}} \times L_{n}^{\alpha, \mathcal{U}}\right) \cap D_{n}\right\} .
$$

Let $\xi \in \omega_{1}$ be such that $K_{n}^{\alpha, \mathcal{U}} \subset O$ for all $n \in F_{\xi}$. Then for every $n \in F_{\beta} \cap F_{\xi}$, we have

$$
\emptyset \neq(O \times V) \cap\left(K_{n}^{\alpha, \mathcal{U}} \times L_{n}^{\alpha, \mathcal{U}}\right) \subset(O \times V) \cap M_{n},
$$

which completes the proof of Lemma 2.5.

Next lemma gives consistent examples of countable spaces $X$ such that $\zeta(X) \leq$ $\omega_{1}$.

Lemma 2.6. In the Miller model, suppose that $X=\langle\omega, \tau\rangle$ is a topological space and $x \in \omega$ is such that $\mathcal{U}=\{U \in \mathcal{P}(\omega): x \in \operatorname{Int}(U)\}$ is Menger. Then $\zeta(X, x) \leq$ $\omega_{1}$.

Proof. For every $n \in \omega$, fix $A_{n}=\left\{a_{k}^{n}: k \in \omega\right\} \subset \omega$ such that $x \in \bar{A}_{n}$. For every $U \in \mathcal{U}$, set

$$
\phi(U)(n)=\min \left\{k: a_{k}^{n} \in U\right\} \text { and } \Phi(U)=\left\{z \in \omega^{\omega}: \forall n(z(n) \leq \phi(\mathcal{U})(n))\right\}
$$

and note that $\Phi$ is a compact-valued map from $\mathcal{U}$ to $\omega^{\omega}$. We claim that it is upper semicontinuous, i.e., for every open $W \subset \omega^{\omega}$ containing $\Phi(U)$ for some $U \in \mathcal{U}$, there exists an open neighborhood $O$ of $U$ in $\mathcal{U}$ such that $\Phi\left(U^{\prime}\right) \subset W$ for all $U^{\prime} \in \mathcal{U} \cap O$. For $U, W$ as above find $m \in \omega$ such that

$$
\Phi(U)=\prod_{n \in \omega}(\phi(U)(n)+1) \subset \prod_{n \leq m}(\phi(U)(n)+1) \times \prod_{n>m} \omega \subset W .
$$

Set $O=\left\{U^{\prime} \in \mathcal{U}: \forall n \leq m\left(\phi\left(U^{\prime}\right)(n) \leq \phi(U)(n)\right)\right\}$ and note that $O$ is open in $\mathcal{P}(\omega)$ and $\Phi\left(U^{\prime}\right) \subset W$ for all $U^{\prime} \in O \cap \mathcal{U}$.

Since $\mathcal{U}$ is Menger and $\Phi$ is compact-valued and upper semicontinuous, $Z:=$ $\bigcup_{U \in \mathcal{U}} \Phi(U) \subset \omega^{\omega}$ is Menger by [22, Lemma 1]. Applying [23, Lemma 2.3], we conclude that there exists $Y \in\left[\omega^{\omega}\right]^{\omega_{1}}$ such that for every $z \in Z$ (in particular, for every $z$ of the form $\phi(U)$, where $U \in \mathcal{U})$ there exists $y \in Y$ such that $z(n) \leq y(n)$ for all $n \in \omega$. It follows from the above that $K_{n}^{y}=\left\{a_{k}^{n}: k \leq y(n)\right\}$, where $y \in Y$ and $n \in \omega$, are witnessing for $\zeta(X, x) \leq \omega_{1}$.

Lemma 2.7. Suppose that $X$ is a Tychonoff space such that $X^{n}$ is Menger for all $n \in \omega$, and $0 \in A \in\left[C_{p}(X)\right]^{\omega}$ is such that 0 is a limit point of $A$. Then $\mathcal{U}=\{U \in \mathcal{P}(A): 0 \in \operatorname{Int}(U)\}$ is Menger as a subspace of $\mathcal{P}(A)$, where the interior is considered in the topology on $A$ inherited from $C_{p}(X)$.

Proof. By the definition of the topology of $C_{p}(X)$ we have that

$$
\mathcal{U}=\bigcup_{n, m \in \omega} \bigcup_{\vec{x}=\left\langle x_{0}, \ldots, x_{n-1}\right\rangle \in X^{n}} \uparrow U_{n, m, \vec{x}},
$$


where $U_{n, m \vec{x}}=\left\{a \in A: \forall i<n\left(a\left(x_{i}\right)<1 / m\right)\right\}$ and $\uparrow B=\left\{B^{\prime} \subset A: B \subset B^{\prime}\right\}$ for all $B \subset A$. In the same way as in Lemma 2.6, we can check that the map

$$
X^{n} \ni \vec{x} \mapsto \uparrow U_{n, m \vec{x}} \subset \mathcal{P}(A)
$$

is compact-valued and upper semicontinuous for all $n, m \in \omega$, and hence by [22, Lemma 1] $\mathcal{U}$ is Menger being a countable union of its Menger subspaces.

Finally, we have all necessary ingredients for the proof of Theorem 1.1. It suffices to prove that in the Miller model the product of any two countable $M$ separable spaces $X, Y$ is $M$-separable, provided that $X$ is a subspace of $C_{p}(Z)$ and $C_{p}(Z)$ is $M$-separable. By [21, Theorem 35], we have that $Z^{n}$ has the Menger property for all $n \in \omega$, and hence for every $x \in X$, the family $\mathcal{U}=\{U \in$ $\mathcal{P}(X): x \in \operatorname{Int}(U)\}$ is Menger as a subspace of $\mathcal{P}(X)$ by Lemma 2.7. Applying Lemma 2.6, we conclude that $\zeta(X) \leq \omega_{1}$, and hence $X$ has property ( $\dagger$ ) by Lemma 2.3. It remains to apply Lemma 2.5.

\section{ACKNOWLEDGEMENTS}

This research was supported by the Slovenian Research Agency grants P10292 and N1-0083 and the Austrian Science Fund FWF grants I 2374-N35 and I $3709-\mathrm{N} 35)$.

\section{REFERENCES}

[1] Arkhangel'skiǔ, A., Hurewicz spaces, analytic sets and fan tightness of function spaces, Dokl. Akad. Nauk SSSR 287 (1986), 525-528. (In Russian)

[2] Barman, D.; Dow, A., Selective separability and SS+, Topology Proc. 37 (2011), 181-204.

[3] Bella, A., Bonanzinga, M., Matveev, M., Variations of selective separability, Topology Appl. 156 (2009), 1241-1252.

[4] Bella, A., Bonanzinga, M., Matveev, M., Tkachuk, V., Selective sepaability: general facts and behaviour in countable spaces, Topology Proc. 32 (2008), 15-30.

[5] Blass, A., Combinatorial cardinal characteristics of the continuum, in: Handbook of Set Theory (M. Foreman, A. Kanamori, and M. Magidor, eds.), Springer, 2010, pp. 395-491.

[6] Blass, A.; Laflamme, C., Consistency results about filters and the number of inequivalent growth types, J. Symbolic Logic 54 (1989), 50-56.

[7] Blass, A.; Mildenberger, H., On the cofinality of ultrapowers, J. Symbolic Logic 64 (1999), 727-736.

[8] Blass, A.; Shelah, S., There may be simple $P_{\aleph_{1}}$ - and $P_{\aleph_{2}}$-points and the Rudin-Keisler ordering may be downward directed, Ann. Pure Appl. Logic 33 (1987), 213-243.

[9] Blass, A.; Shelah, S., Near coherence of filters. III. A simplified consistency proof, Notre Dame J. Formal Logic 30 (1989), 530-538.

[10] Gruenhage, G.; Sakai, M., Selective separability and its variations, Topology Appl. 158 (2011) 1352-1359.

[11] Hurewicz, W., Über die Verallgemeinerung des Borellschen Theorems, Math. Z. 24 (1925), 401-421.

[12] Laflamme, C., Equivalence of families of functions on the natural numbers, Trans. Amer. Math. Soc. 330 (1992), 307-319.

[13] Laver, R., On the consistency of Borel's conjecture, Acta Math. 137 (1976), 151-169.

[14] Menger, K., Einige Überdeckungssätze der Punktmengenlehre, Sitzungsberichte. Abt. 2a, Mathematik, Astronomie, Physik, Meteorologie und Mechanik (Wiener Akademie) 133 (1924), 421-444. 
[15] Miller, A., Rational perfect set forcing, in: Axiomatic Set Theory (J. Baumgartner, D. A. Martin, S. Shelah, eds.), Contemporary Mathematics 31, American Mathematical Society, Providence, Rhode Island, 1984, pp.143-159.

[16] Miller, A.W.; Tsaban, B.; Zdomskyy, L., Selective covering properties of product spaces, II: gamma spaces, Trans. Amer. Math. Soc. 368 (2016), 2865-2889.

[17] Repovš, D.; Zdomskyy, L., On M-separability of countable spaces and function spaces, Topology Appl. 157 (2010), 2538-2541.

[18] Repovš, D.; Zdomskyy, L., Products of Hurewicz spaces in the Laver model, Bull. Symb. Log. 23 (2017), 324-333.

[19] Repovš, D.; Zdomskyy, L., Products of H-separable spaces in the Laver model, Topology Appl. 239 (2018), 115-119.

[20] Sakai, M., Special subsets of reals characterizing local properties of function spaces, In: Selection Principles and Covering Properties in Topology (L. Kočinac, ed.), Quaderni di Matematica 18, Seconda Universita di Napoli, Caserta 2006, 195-225

[21] Scheepers, M., Combinatorics of open covers. VI. Selectors for sequences of dense sets, Quaest. Math. 22 (1999), 109-130.

[22] Zdomskyy, L, A semifilter approach to selection principles, Comment. Math. Univ. Carolin. 46 (2005), 525-539.

[23] Zdomskyy, L., Products of Menger spaces in the Miller model, Adv. Math. 335 (2018), 170-179.

[24] Zenor, P., Hereditary $\mathfrak{m}$-separability and the hereditary $\mathfrak{m}$-Lindelöf property in product spaces and function spaces, Fund. Math. 106 (1980), 175-180.

Faculty of Education, and Faculty of Mathematics and Physics, University of Ljubljana, \& Institute of Mathematics, Physics and Mechanics, 1000 Ljubljana, SLOVENIA.

Email address: dusan.repovs@guest.arnes.si

Institut für Diskrete Mathematik und Geometrie, Technische Universität Wien, Wiedner Hauptstrasse 8-10/104, 1040 Wien, Austria.

Email address: lzdomsky@gmail.com 